\title{
Associação entre a qualidade de vida relacionada à saúde bucal e a capacidade para o trabalho de técnicos administrativos em educação: um estudo transversal
}

\author{
Association between oral health-related quality of life and \\ work ability of technical-administrative in education: \\ a cross-sectional study
}

\author{
Pamella Valente Palma ${ }^{1}$ (D), Isabel Cristina Gonçalves Leite ${ }^{2}$ (D), \\ Rosangela Maria Greco ${ }^{3}$
}

\begin{abstract}
Resumo
Introdução: Trabalhadores ativos constituem uma parcela importante da população que demanda os serviços de saúde. O estudo da relação entre saúde bucal e trabalho visa à melhoria da qualidade de vida e do desempenho profissional. Objetivo: associar a capacidade para o trabalho com a qualidade de vida relacionada à saúde bucal de técnicos administrativos em educação de uma instituição de ensino superior de Minas Gerais. Método: estudo transversal com 833 funcionários, com os quais foram coletados dados de identificação, socioeconômicos, demográficos, autopercepção e morbidade em saúde bucal. O impacto da saúde bucal na qualidade de vida foi avaliado pelo Oral Health Impact Profile (OHIP-14), e a capacidade de trabalho, pelo Índice de Capacidade para o Trabalho (ICT). Os dados foram analisados pelos testes Mann-Whitney e coeficiente de correlação de Spearman. Resultados: $83 \%$ dos entrevistados possuíam ótima ou boa capacidade para o trabalho. Houve correlação positiva e significativa da autopercepção da saúde bucal com a autopercepção da saúde geral $(p<0,001)$ e negativa com o ICT $(p=0,026)$. $\mathrm{Na}$ análise de regressão, ICT total permaneceu significativo para o domínio dor física do OHIP-14. Conclusão: capacidade para o trabalho foi associada ao domínio dor física. Condições sociodemográficas e de autopercepção também impactaram na qualidade de vida associada à saúde bucal.
\end{abstract}

Palavras-chave: saúde bucal; qualidade de vida; saúde do trabalhador; avaliação da capacidade de trabalho; odontologia do trabalho.

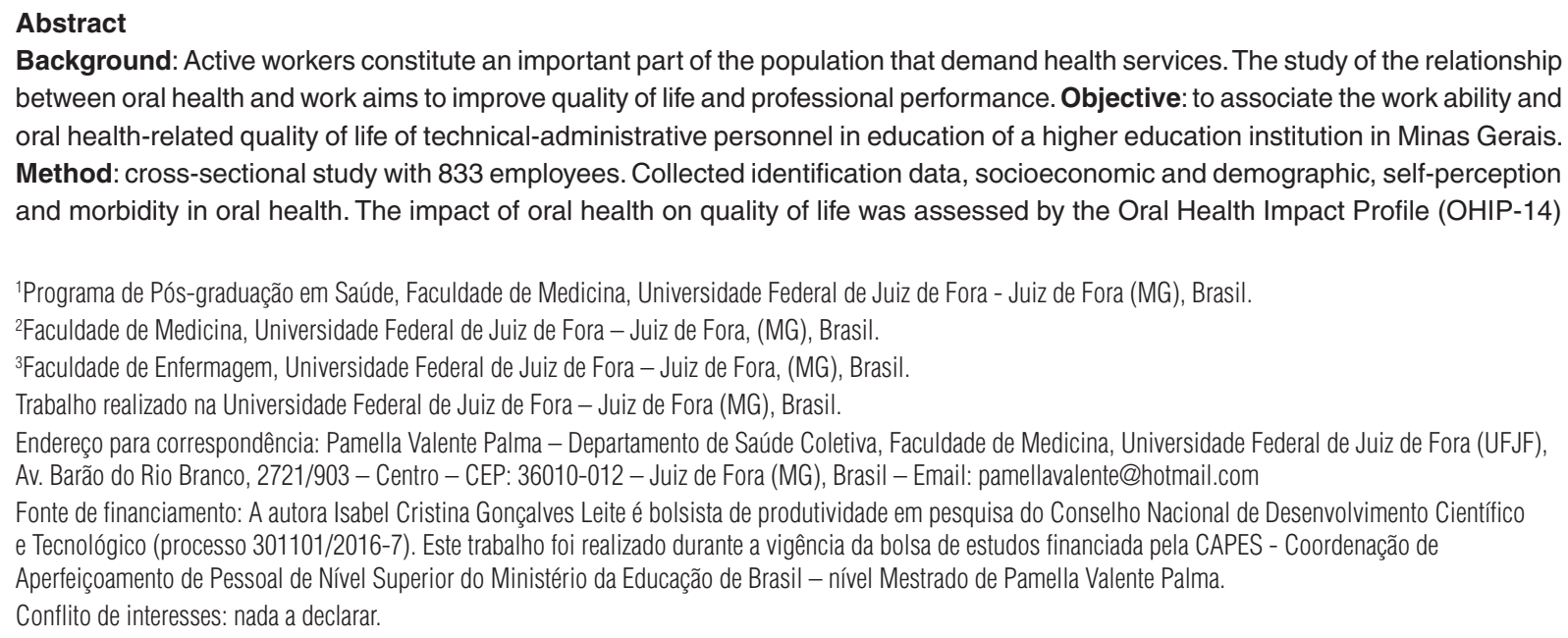

Background: Active workers constitute an important part of the population that demand health services. The study of the relationship between oral health and work aims to improve quality of life and professional performance. Objective: to associate the work ability and oral health-related quality of life of technical-administrative personnel in education of a higher education institution in Minas Gerais. Method: cross-sectional study with 833 employees. Collected identification data, socioeconomic and demographic, self-perception and morbidity in oral health. The impact of oral health on quality of life was assessed by the Oral Health Impact Profile (OHIP-14) 
and the ability to work by the Work Capability Index (WAI). Data analyzed by the Mann-Whitney test and Spearman's correlation coefficient. Results: $83 \%$ of respondents had great or good ability to work. Positive and significant correlation of self-perception of oral health with self-perception of general health $(p<0.001)$, and negative correlation with CTI $(p=0.026)$. In the regression analysis, total WAI remained significant for the physical pain domain of OHIP-14. Conclusion: ability to work was associated with the physical pain domain. Sociodemographic and self-perception conditions also impacted the quality of life associated with oral health. Keywords: oral health; quality of life; occupational health; work capacity evaluation; occupational dentistry.

\section{INTRODUÇÃO}

O estudo da relação entre saúde bucal e trabalho trata de promover, preservar e recuperar a saúde bucal de populações inseridas nos mais diversos tipos de trabalho, o que contribui para a melhora de sua qualidade de vida e de trabalho'1. É necessária a análise da população adulta economicamente ativa, exposta não só aos principais fatores etiológicos comuns das doenças bucais, mas também aos riscos do próprio ambiente de trabalho ${ }^{2}$.

A perda da capacidade do trabalho está diretamente relacionada aos determinantes do processo saúde-doença, implicando o perfil dos trabalhadores, o quadro epidemiológico e as práticas de saúde voltadas para essa parcela da população ${ }^{2}$. Sendo assim, a capacidade de trabalho pode estar associada com alguns fatores, como a idade, o sexo, o nível socioeconômico, o grau de escolaridade e o estilo de vida (fumo, ingestão de bebidas alcoólicas e prática de atividade física) ${ }^{3}$.

A Organização Mundial de Saúde, em 1995, definiu a qualidade de vida como sendo

[...] a percepção do indivíduo de sua posição na vida no contexto da cultura e sistema de valores nos quais ele vive e em relação aos seus objetivos, expectativas, padrões e preocupações ${ }^{4}$.

Os indicadores subjetivos têm sido cada vez mais utilizados nas pesquisas, valorizando as dimensões sociais da saúde, em vez de enfatizar somente os indicadores clínicos. Essa abordagem, extensiva à área da saúde bucal, permite planejar políticas públicas e melhorar o acesso aos serviços ${ }^{5}$.

Entretanto, os estudos na literatura sobre a qualidade de vida relacionada à saúde, realizados na população geral de adultos que não apresentam nenhuma patologia específica, são insuficientes. A maioria da população é formada por adultos que necessitam de serviços de saúde; são trabalhadores ativos que possuem peculiaridades epidemiológicas. Dessa forma, sua condição de saúde e de bem-estar, além de alterações no padrão de comportamento e estilo de vida, pode proporcionar um impacto econômico e social ${ }^{6}$.

Desse modo, este estudo tem por objetivo associar a capacidade para o trabalho com a qualidade de vida relacionada à saúde bucal de técnicos administrativos em educação de uma instituição de ensino superior de Minas Gerais.

\section{MÉTODO}

Trata-se de um estudo transversal, realizado por censo com abordagem da categoria dos técnicos administrativos da Universidade Federal de Juiz de Fora (UFJF), uma universidade pública sediada em Juiz de Fora (MG), com um campus avançado em Governador Valadares (MG). A instituição posiciona-se como um polo científico e cultural de uma região de mais de 3 milhões de habitantes. O público-alvo deste estudo são os servidores ocupantes do cargo de técnicos em administração, em um total de 1.291 funcionários, quando da pesquisa. Desse total, o assistente em administração totaliza 424 servidores $(32,8 \%)$. A atuação deles acontece em unidades acadêmicas e administrativas e representa a diversidade dos ambientes de trabalho dessa instituição.

O cargo de técnico em administração é uma função na qual a exigência de escolaridade é diversa, havendo servidores no nível A (ensino fundamental incompleto, cargos em extinção) até nível $\mathrm{E}$ (nível superior completo). As atividades desse cargo concentram-se em dar suporte administrativo e técnico nas diferentes áreas da universidade e, inclusive, assessorar as atividades de ensino, pesquisa e extensão ${ }^{7}$.

Foram incluídos aqueles funcionários técnico administrativos efetivos da universidade, em exercício ativo da função. Foram excluídos os que estavam em situação de afastamento do trabalho por qualquer motivo de licença ou que tivessem sido cedidos a outra instituição ou transferidos de outro órgão público.

Para a coleta de dados, todos os pesquisadores foram treinados previamente. O instrumento de coleta foi o mesmo utilizado em um estudo prévio ${ }^{8}$ relativo ao "I Inquérito sobre condições de trabalho e de vida dos Trabalhadores Técnico-Administrativos em Educação da Universidade Federal de Juiz de Fora”. No presente estudo, foram utilizados os dados sobre autopercepção de saúde geral, morbidade em saúde bucal e de autopercepção em saúde bucal, qualidade de vida em saúde bucal, Índice de Capacidade para o Trabalho (ICT), dados de identificação e socioeconômicos.

Todos os sujeitos na amostra responderam ao Oral Health Impact Profile (OHIP-14), desenvolvido por Slade e Spencer, em 1994, em versão validada e adaptada para o português do Brasil $1^{9}$. O questionário proposto mede a limitação, o desconforto e a incapacidade atribuída à condição bucal.

O questionário (OHIP), traduzido para o português como "Perfil do Impacto da Saúde Oral", integra duas perguntas para cada uma das sete dimensões: limitação funcional, dor física, desconforto psicológico, incapacidade física, incapacidade psicológica, incapacidade social e desvantagem social.

O escore final do OHIP-14 total pode alcançar 70 pontos (cada domínio apresenta duas questões, cada qual com pontuação 
máxima de 5 pontos, portanto 10 por domínio, de um total de 7 domínios). Para a análise descritiva do OHIP, para cada dimensão, este teve suas respostas dicotomizadas: com impacto para as respostas "frequentemente" e "sempre" e sem impacto para as respostas "às vezes", "raramente" e "nunca".

As questões estão organizadas de modo que os participantes indiquem, segundo uma escala tipo de Likert contendo cinco categorias de resposta, com que frequência experienciaram cada um dos problemas, dentro de um período de referência de 12 meses. As categorias de resposta e respectivas cotações são: quase sempre $=5$; algumas vezes $=4$; poucas vezes $=3$; raramente $=2 ;$ nunca $=1$.

O ICT é um instrumento traduzido, adaptado e validado para o Brasil, em forma de questionário, com questões objetivas para serem respondidas pelo próprio trabalhador ${ }^{10}$. Para responder às questões, é importante que este possua escolaridade mínima da quarta série do ensino fundamental, recomendação dada por viabilizar a melhor compreensão das questões. O instrumento é formado por sete itens, cada um com uma ou mais questões. O índice de cada indivíduo varia de um escore de 7 a 49 pontos e é determinado com base nas respostas dadas às várias questões sobre as exigências físicas e mentais do trabalho, o estado de saúde e os recursos do trabalhador ${ }^{11}$.

No presente estudo, o ICT foi dicotomizado ${ }^{8} \mathrm{em}$ : reduzida capacidade para o trabalho ( 7 a 36 pontos) e boa capacidade para o trabalho (37 a 49 pontos).

Os dados foram digitados no programa SPSS 19.0 e depois foram submetidos a testes estatísticos: no caso de variáveis contínuas, o padrão de normalidade foi avaliado pelo teste Kolmogorov-Smirnov e indicou que a distribuição dos dados não seguia padrão de normalidade, por isso foi utilizado um teste não paramétrico (Mann-Whitney para variáveis dicotômicas). Para analisar a correlação entre OHIP-14 e ICT, o coeficiente de correlação de Spearman foi usado. Foi realizada regressão linear múltipla reunindo aquelas variáveis que foram significativas $(\mathrm{p}<0,05)$ na análise bivariada, tendo o OHIP-total como variável dependente, e o ICT, como variável independente, controlado por características socioeconômicas e demográficas, autopercepção de saúde bucal e necessidade de tratamento. A multicolinearidade entre as variáveis foi avaliada pelo Variance Inflation Factor (VIF). O nível de significância estatística admitido foi de $5 \%$.

Todos os pacientes elegíveis foram convidados a participar e, ao concordarem, assinaram o Termo de Consentimento Livre e Esclarecido. A presente pesquisa foi submetida ao Comitê de Ética em Pesquisas com Seres Humanos da UFJF (Parecer no 224/2010) e aprovada por ele.

\section{RESULTADOS}

Os dados foram coletados de junho de 2013 a agosto de 2014. A população-alvo do estudo era composta por 1.291 funcionários, dos quais 102 se enquadraram nos critérios de exclusão. Então, foram abordados 1.189 indivíduos, mas 95 se recusaram a participar e 261 não retornaram com o instrumento, totalizando, assim, uma população de estudo de 833 funcionários. A análise dos dados mostrou boa confiabilidade interna do OHIP (alfa de Cronbach $=0,92$ ).

A amostra de técnicos administrativos foi constituída predominantemente por homens $(51,5 \%)$, brancos $(67 \%)$, casados ou em união estável (62,7\%), com média de idade de 45,1 anos (desvio-padrão =11). Quanto à escolaridade, a maioria possuía pós-graduação concluída (54,4\%). Em relação à renda familiar, $45,8 \%$ tinham renda familiar entre $\mathrm{R} \$ 3.940,00$ e $\mathrm{R} \$ 7.880,00$.

No que se refere à autopercepção sobre seu estado de saúde geral e bucal, $51,8 \%$ e $50 \%$ dos entrevistados, respectivamente, classificaram como boa. Além disso, 52,6\% afirmaram necessitar de tratamento atualmente. Sobre a presença de odontalgia nos últimos seis meses, $84,3 \%$ afirmaram que não apresentaram episódios. A maioria dos técnicos administrativos relatou visita ao cirurgião-dentista a menos de um ano $(64,6 \%)$, para check-up (49,7\%) e ter utilizado o serviço particular (73,7\%). Com relação à situação da dentição autorreferida, 90,4\% afirmaram ser dentados superior e inferior.

Quanto à classificação do ICT, 40\% dos entrevistados apresentaram ótima capacidade para o trabalho.

Foi verificada correlação positiva e significativa da autopercepção da saúde bucal com a autopercepção da saúde geral $(p<0,001)$ e negativa da autopercepção da saúde bucal com o ICT $(\mathrm{p}=0,026)$.

Em relação à frequência de impacto de cada domínio do OHIP-14 (Tabela 1), os domínios dor física (6,5\%) e desconforto psicológico $(6,3 \%)$ apresentaram o maior impacto.

O presente estudo não encontrou associação do OHIP-14 total com sexo $(\mathrm{p}=0,435)$ ou com estado civil $(\mathrm{p}=0,270)$. As variáveis idade, cor da pele autodeclarada e escolaridade foram estatisticamente significativas tanto para o OHIP-14 total ( $\mathrm{p}<0,001)$ como para todos os domínios $(\mathrm{p}<0,05)$ (Tabela 2).

Tabela 1. Distribuição dos técnicos administrativos em educação da Universidade Federal de Juiz de Fora, de acordo com a frequência do impacto, por domínios do OHIP-14, Juiz de Fora, 2018

\begin{tabular}{lcc} 
Dimensão de saúde bucal & $\begin{array}{c}\text { Com impacto n } \\
(\mathbf{\%})\end{array}$ & $\begin{array}{c}\text { Sem impacto n } \\
(\mathbf{\%})\end{array}$ \\
\hline Limitação funcional & $20(2,4)$ & $803(97,6)$ \\
Dor física & $54(6,5)$ & $771(93,5)$ \\
Desconforto psicológico & $52(6,3)$ & $773(93,7)$ \\
Incapacidade física & $23(2,8)$ & $801(97,2)$ \\
Incapacidade psicológica & $30(3,6)$ & $795(96,4)$ \\
Incapacidade social & $8(1,0)$ & $816(99,0)$ \\
Desvantagem social & $9(1,1)$ & $814(98,9)$ \\
\hline
\end{tabular}

Nota: OHIP = Oral Health Impact Profile 
Tabela 2. Média, desvio-padrão e p-valor (Mann-Whitney) das variáveis sociodemográficas, por domínios e para OHIP-14 total dos técnicos administrativos em educação da Universidade Federal de Juiz de Fora, Juiz de Fora, 2018

\begin{tabular}{|c|c|c|c|c|c|c|c|c|}
\hline \multirow{2}{*}{ Variável ${ }^{\star}$} & \multicolumn{7}{|c|}{ Médias por domínio do OHIP (dp) } & \multirow{2}{*}{ Total } \\
\hline & 1 & 2 & 3 & 4 & 5 & 6 & 7 & \\
\hline \multicolumn{9}{|l|}{ Sexo } \\
\hline \multirow[t]{2}{*}{ Masculino } & 9,48 & 8,78 & 8,93 & 9,35 & 9,24 & 9,63 & 9,62 & 65,03 \\
\hline & $(1,39)$ & $(1,61)$ & $(1,68)$ & $(1,44)$ & $(1,42)$ & $(1,21)$ & $(1,15)$ & $(8,30)$ \\
\hline \multirow[t]{2}{*}{ Feminino } & 9,46 & 8,66 & 8,69 & 9,28 & 9,12 & 9,63 & 9,62 & 64,46 \\
\hline & $(1,60)$ & $(1,88)$ & $(1,93)$ & $(1,60)$ & $(1,62)$ & $(1,39)$ & $(1,42)$ & $(10,0)$ \\
\hline p-valor & 0,623 & 0,931 & 0,090 & 1,00 & 0,408 & 0,252 & 0,031 & 0,435 \\
\hline \multicolumn{9}{|l|}{ Idade } \\
\hline \multirow[t]{2}{*}{$\leq 45$ anos } & 9,78 & 8,93 & 9,12 & 9,57 & 9,43 & 9,81 & 9,79 & 66,43 \\
\hline & $(0,78)$ & $(1,44)$ & $(1,40)$ & $(1,09)$ & $(1,08)$ & $(0,79)$ & $(0,94)$ & $(5,91)$ \\
\hline \multirow[t]{2}{*}{$>45$ anos } & 9,22 & 8,55 & 8,57 & 9,10 & 8,98 & 9,48 & 9,48 & 63,39 \\
\hline & $(1,85)$ & $(1,94)$ & $(2,05)$ & $(1,77)$ & $(1,78)$ & $(1,59)$ & $(1,50)$ & $(10,95)$ \\
\hline p-valor & $<0,001$ & 0,009 & $<0,001$ & $<0,001$ & 0,001 & 0,002 & $<0,001$ & $<0,001$ \\
\hline \multicolumn{9}{|l|}{ Estado civil } \\
\hline \multirow[t]{2}{*}{ Solteiro/viúvo/divorciado } & 9,37 & 8,49 & 8,65 & 9,19 & 9,09 & 9,57 & 9,57 & 63,93 \\
\hline & $(1,85)$ & $(2,04)$ & $(2,03)$ & $(1,80)$ & $(1,70)$ & $(1,57)$ & $(1,54)$ & $(10,96)$ \\
\hline \multirow[t]{2}{*}{ Casado ou união estável } & 9,49 & 8,83 & 8,89 & 9,37 & 9,23 & 9,65 & 9,61 & 65,09 \\
\hline & $(1,31)$ & $(1,55)$ & $(1,67)$ & $(1,35)$ & $(1,39)$ & $(1,13)$ & $(1,17)$ & $(8,01)$ \\
\hline p-valor & 0,817 & 0,032 & 0,267 & 0,505 & 0,349 & 0,561 & 0,532 & 0,270 \\
\hline \multicolumn{9}{|l|}{ Cor da pele autodeclarada } \\
\hline \multirow[t]{2}{*}{ Branca } & 9,64 & 8,91 & 9,00 & 9,48 & 9,33 & 9,77 & 9,76 & 65,89 \\
\hline & $(1,09)$ & $(1,49)$ & $(1,54)$ & $(1,16)$ & $(1,21)$ & $(0,88)$ & $(0,80)$ & $(6,72)$ \\
\hline \multirow[t]{2}{*}{ Não branca } & 9,13 & 8,33 & 8,42 & 8,98 & 8,88 & 9,34 & 9,32 & 62,41 \\
\hline & $(2,06)$ & $(2,13)$ & $(2,21)$ & $(2,03)$ & $(1,99)$ & $(1,86)$ & $(1,91)$ & $(12,49)$ \\
\hline p-valor & $<0,001$ & 0,001 & $<0,001$ & 0,001 & 0,011 & $<0,001$ & 0,003 & $<0,001$ \\
\hline \multicolumn{9}{|l|}{ Escolaridade ${ }^{*}$} \\
\hline \multirow[t]{2}{*}{ Até médio completo } & 8,88 & 8,29 & 8,42 & 8,92 & 8,85 & 9,22 & 9,18 & 61,75 \\
\hline & $(2,45)$ & $(2,56)$ & $(2,27)$ & $(2,19)$ & $(2,11)$ & $(2,12)$ & $(2,13)$ & $(13,79)$ \\
\hline \multirow[t]{2}{*}{ Superior + pós-graduação } & 9,64 & 8,84 & 8,93 & 9,43 & 9,28 & 9,74 & 9,74 & 65,61 \\
\hline & $(1,02)$ & $(1,55)$ & $(1,64)$ & $(1,24)$ & $(1,29)$ & $(0,91)$ & $(0,88)$ & $(7,12)$ \\
\hline p-valor & $<0,001$ & 0,004 & 0,006 & 0,001 & 0,053 & 0,001 & $<0,001$ & $<0,001$ \\
\hline
\end{tabular}

${ }^{*}$ Excluídos não respondentes $($ sexo $=7$; escolaridade $=9$; estado civil $=13$; cor de pele $=11$; idade $=29)$

Nota: OHIP = Oral Health Impact Profile. $\mathrm{dp}=$ desvio padrão

As variáveis saúde geral autopercebida, saúde bucal autopercebida e necessidade percebida de tratamento odontológico foram estatisticamente significativas com o OHIP-14 total ( $\mathrm{p}<0,001$ para cada uma dessas variáveis) e com todos os domínios ( $\mathrm{p}<0,01)$ (Tabela 3).

Sobre o ICT, não foi encontrada associação significativa neste estudo com o OHIP-14 total, mas, nos domínios dor física, desconforto psicológico e desvantagem social, é possível observar essa associação $(\mathrm{p}<0,05)$.

A Tabela 4 apresenta o resultado da análise de regressão. A respeito do domínio dor física, as variáveis que permaneceram significativas após o ajuste foram: cor da pele, estado civil, autopercepção de saúde bucal, necessidade percebida de tratamento odontológico atualmente e ICT total. Essas variáveis justificam $48 \%$ da variabilidade desse domínio.

Para o domínio desconforto psicológico, após o ajuste, as variáveis que se mantiveram significativas foram cor da pele, autopercepção de saúde bucal e necessidade percebida de tratamento odontológico atualmente, que explicam quase $50 \%$ da variabilidade desse item.

Escolaridade, idade, cor da pele e autopercepção de saúde bucal continuaram significativas em relação ao domínio desvantagem social. Esse modelo traduz $32 \%$ da variabilidade do referido domínio. 
Tabela 3. Média e p-valor (Mann-Whitney) das variáveis de autopercepção, por domínios e para OHIP-14 total dos técnicos administrativos em educação da Universidade Federal de Juiz de Fora, Juiz de Fora, 2018

Variável $^{*}$

\section{Saúde geral autopercebida}

Muito boa + boa

Moderada a ruim

p-valor

Saúde bucal autopercebida

Muito boa + boa

Moderada a ruim

p-valor

Necessidade percebida de tratamento odontológico atualmente

Sim

Não

p-valor

ICT

Ótimo + bom

Moderado + baixo

p-valor

1

9,56

$(1,32)$

8,99

$(2,03)$

0,008

$(1,60)$

7,91

$(2,00)$

$<0,001$

$$
9,72
$$

$(1,00)$

8,75

$(2,18)$

$<0,001$

9,07

$(1,42)$

7,68

$(1,98)$

$<0,001$

$$
9,28
$$

$(1,66)$

$$
9,71
$$

$(1,12)$

$<0,001$

8,23

$(1,88)$

9,28

$(1,21)$

9,43

$(1,59)$

9,48

$(1,46)$

0,566

8,87

$(1,86)$

8,01

$(1,31)$

0,048
$<0,001<0,001$

Médias por domínio do OHIP (dp)

3

4

8,92

$(1,67)$

9,41

$(1,36)$

8,03

$(2,35)$

$<\mathbf{0 , 0 0 1}$

8,63

$(2,18)$

$<0,001$

5

\section{9,22}

$(1,41)$

7,52

$(2,21)$

$<0,001$

\section{8,31}

$(2,05)$

9,36

$(1,26)$

$<0,001$

8,78

$(1,90)$

8,00
9,57

$(1,19)$

8,52

$(2,04)$

$<0,001$

\section{9,26}

$(1,43)$

8,68

$(1,89)$

$<0,001$

\section{9,49}

$(1,21)$

8,26

$(1,91)$

$<0,001$

$(0,99)$

$<0,001$

$(1,62)$

0,036

9,26
$(1,66)$
9,41
$(1,15)$
0,631

9,01

$(1,81)$

9,65

\section{8,82}

$(1,78)$

9,60

$(0,97)$

$<0,001$

\section{9,12}

$(1,65)$

9,30

$(1,18)$

0,631

0,479

9,69
$(1,16)$
9,27
$(1,74)$
$\mathbf{0 , 0 0 1}$

7

Total

${ }^{\star}$ Excluídos não respondentes (autopercepção de saúde geral $=1$; autopercepção de saúde bucal $=11$; necessidade de tratamento $=12 ; \mathrm{ICT}=45$ )

Nota: OHIP $=$ Oral Health Impact Profile. ICT $=$ Índice de Capacidade para o Trabalho. $\mathrm{dp}=$ desvio padrão

\begin{tabular}{|c|c|c|c|c|c|c|c|c|c|c|c|c|}
\hline & \multicolumn{3}{|c|}{ OHIP-14 } & \multicolumn{3}{|c|}{ Dor física } & \multicolumn{3}{|c|}{$\begin{array}{l}\text { Desconforto } \\
\text { psicológico }\end{array}$} & \multicolumn{3}{|c|}{ Desvantagem social } \\
\hline $\mathrm{r}^{2}$ ajustado & \multicolumn{3}{|c|}{0,312} & \multicolumn{3}{|c|}{0,482} & \multicolumn{3}{|c|}{0,496} & \multicolumn{3}{|c|}{0,325} \\
\hline Variáveis & $\mathrm{B}$ & IC 95\% & $\mathrm{p}$ & $\beta$ & IC 95\% & $\mathrm{p}$ & $\mathrm{B}$ & IC $95 \%$ & $\mathrm{p}$ & $\beta$ & IC 95\% & $\mathrm{p}$ \\
\hline Sexo (masculino) & & & & & & & & & & $-0,17$ & $\begin{array}{c}-0,36 \\
0,02\end{array}$ & 0,081 \\
\hline Escolaridade (fundamental incompleto) & 0,07 & $\begin{array}{c}-0,003 \\
0,78\end{array}$ & 0,052 & 0,05 & $\begin{array}{c}-0,02 \\
0,12\end{array}$ & 0,182 & 0,04 & $\begin{array}{c}-0,04 \\
0,11\end{array}$ & 0,308 & 0,11 & $\begin{array}{l}0,06 \\
0,17\end{array}$ & $<0,001$ \\
\hline Idade & $-0,07$ & $\begin{array}{l}-0,11 \\
-0,001\end{array}$ & 0,048 & $-0,01$ & $\begin{array}{c}-0,02 \\
0,01\end{array}$ & 0,179 & $-0,01$ & $\begin{array}{c}-0,02 \\
0,01\end{array}$ & 0,288 & $-0,01$ & $\begin{array}{l}-0,01 \\
-0,00\end{array}$ & 0,046 \\
\hline Cor da pele (branco) & $-0,10$ & $\begin{array}{l}-3,31 \\
-0,78\end{array}$ & 0,002 & $-0,28$ & $\begin{array}{l}-0,51 \\
-0,06\end{array}$ & 0,013 & $-0,32$ & $\begin{array}{l}-0,56 \\
-0,07\end{array}$ & 0,012 & $-0,29$ & $\begin{array}{l}-0,48 \\
-0,09\end{array}$ & 0,004 \\
\hline Estado civil (casado ou em união estável) & & & & $-0,24$ & $\begin{array}{l}-0,46 \\
-0,02\end{array}$ & 0,034 & & & & & & \\
\hline $\begin{array}{l}\text { Autopercepção de saúde geral } \\
\text { (muito boa) }\end{array}$ & $-0,47$ & $\begin{array}{c}-1,28 \\
0,34\end{array}$ & 0,253 & $-0,12$ & $\begin{array}{c}-0,28 \\
0,03\end{array}$ & 0,122 & $-0,13$ & $\begin{array}{c}-0,30 \\
0,05\end{array}$ & 0,153 & $-0,08$ & $\begin{array}{l}-0,22 \\
0,06\end{array}$ & 0,274 \\
\hline $\begin{array}{l}\text { Autopercepção de saúde bucal } \\
\text { (muito boa) }\end{array}$ & $-0,40$ & $\begin{array}{l}-5,21 \\
-3,73\end{array}$ & $<0,001$ & $-0,70$ & $\begin{array}{l}-0,86 \\
-0,55\end{array}$ & $<0,001$ & $-0,99$ & $\begin{array}{l}-1,14 \\
-0,84\end{array}$ & $<0,001$ & $-0,33$ & $\begin{array}{l}-0,45 \\
-0,21\end{array}$ & $<0,001$ \\
\hline $\begin{array}{l}\text { Necessidade percebida de tratamento } \\
\text { odontológico atualmente } \\
(\operatorname{sim})\end{array}$ & 1,36 & $\begin{array}{l}0,18 \\
2,53\end{array}$ & 0,024 & 0,46 & 0,23 & $<0,001$ & 0,34 & $\begin{array}{l}0,09 \\
0,58\end{array}$ & 0,007 & 0,15 & $-0,04$ & 0,128 \\
\hline ICT total & $-0,06$ & $\begin{array}{c}-0,21 \\
0,01\end{array}$ & 0,081 & 0,02 & $\begin{array}{l}0,01 \\
0,03\end{array}$ & 0,051 & 0,01 & $\begin{array}{c}-0,02 \\
0,02\end{array}$ & 0,057 & 0,01 & $\begin{array}{c}-0,03 \\
0,04\end{array}$ & 0,087 \\
\hline
\end{tabular}

Tabela 4. Modelo de regressão linear múltipla: preditores do OHIP dos técnicos administrativos em educação da Universidade Federal de Juiz de Fora, 2018

Nota: OHIP = Oral Health Impact Profile. ICT = Índice de Capacidade para o Trabalho 


\section{DISCUSSÃO}

Este estudo associou a qualidade de vida relacionada à saúde bucal com a capacidade para o trabalho de técnicos administrativos em educação de uma instituição superior de ensino em Minas Gerais. O ICT manteve-se associado ao OHIP no domínio dor física, controlado por variáveis sociodemográficas e de autopercepção. Características de cor de pele, escolaridade, idade, autopercepção de saúde bucal e necessidade percebida de tratamento odontológico permaneceram no modelo final para o OHIP-14.

Como limitações do estudo, tem-se uma taxa de resposta de $70 \%$ da população proveniente das perdas decorrentes da falta de retorno do questionário por alguns entrevistados. A análise do perfil dos que não devolveram os questionários é semelhante ao dos incluídos no estudo no que se refere a variáveis demográficas e níveis de ocupação. Uma vez que as percepções de saúde e de desvantagem social são influenciadas pelo contexto social, cultural e político em que estão inseridas, é possível que essa amostra de trabalhadores não reflita a população adulta brasileira, já que se trata de uma amostra com alto nível de escolaridade, bom padrão socioeconômico e acesso aos serviços de saúde, diferente do padrão habitual de um trabalhador brasileiro ${ }^{12}$. No entanto, os resultados apontam aspectos importantes no contexto do universo no qual foram obtidos, qual seja, servidores públicos com atividades-meio vinculados à área da educação.

No presente estudo, a prevalência do sexo masculino foi de $51,5 \%$, o que foi semelhante a outros estudos com trabalhadores do setor industriário ${ }^{13-15}$. Os técnicos estudados apresentavam bons hábitos de higiene, e destacou-se o padrão de uso de flúor condizente com o preconizado na atualidade ${ }^{16}$.

Sobre a variável referente à necessidade percebida de tratamento, a maior parte da população relatava necessitar de tratamento odontológico atualmente, embora tenha relatado visita ao cirurgião-dentista a menos de um ano, no serviço particular e por motivo de check-up. Essa característica é corroborada por outro estudo transversal com amostras representativas das populações adultas americana e australiana ${ }^{17}$. O padrão diferenciado de acesso a serviços pode ser justificado pelo alto nível socioeconômico dos entrevistados de ambos os estudos ${ }^{12}$. Na literatura, também se observa a relação entre a desigualdade social e a saúde bucal e conclui-se que indivíduos que vivem em áreas de privação possuem experiências, percepções e comportamentos mais negativos em saúde quando comparados àqueles de áreas mais privilegiadas ${ }^{18}$.

Sobre a diferença do gênero em relação ao impacto da saúde bucal na qualidade de vida, foi observado maior impacto no sexo feminino, o que condiz com os resultados da literatura ${ }^{19}$. Isso pode ser justificado pelo fato de as mulheres terem uma capacidade maior na percepção dos agravos e nas limitações impostas por eles. As diferenças cognitivas, biológicas e psicológicas entre homens e mulheres são relacionadas à construção social e psicológica, intimamente conectadas ${ }^{20}$.

Da mesma maneira como no presente estudo, associação independente da escolaridade com o OHIP foi identificada em trabalhadores administrativos de uma indústria têxtil de São Paulo $^{21}$. Esse estudo identificou que, após ajuste pela capacidade para trabalho e nível de escolaridade, as variáveis sexo e idade perderam sua significância.

A autopercepção de saúde bucal e geral foi alta no presente estudo, caracterizado por uma amostra com média de idade superior a 45 anos, brancos e com pós-graduação. Indivíduos americanos não brancos tiveram pior autopercepção de saúde bucal, maiores sintomas e problemas relacionados aos dentes do que os declarados brancos. Além disso, quanto mais jovem e com menor nível de escolaridade, percebeu-se maior negligência bucal acumulada, e, dessa forma, os sujeitos dariam menor prioridade à saúde bucal ${ }^{22}$. Por outro lado, quanto mais velho é um trabalhador, as exigências do trabalho tendem a ser mais percebidas, podendo afetar diretamente sua saúde e sua produtividade no ambiente de trabalho ${ }^{23}$. Da mesma forma, um estudo canadense apontou que a faixa etária de 45 a 65 anos tem piores indicadores de qualidade de vida relacionada à saúde bucal em comparação com os idosos ${ }^{24}$.

A autopercepção da saúde bucal foi correlacionada positivamente e de forma significativa com a autopercepção de saúde geral, assim como outros estudos ${ }^{8,12}$. Também se correlacionou com o ICT, semelhante a estudos prévios com industriários ${ }^{13,21}$. Essa associação entre melhor condição da capacidade para o trabalho e melhor qualidade da saúde, tanto física quanto mental, evidencia a integralidade da saúde ${ }^{25}$.

O desgaste decorrente das exigências do trabalho desencadeia respostas fisiológicas crônicas e agudas, reações psicológicas e comportamentais, reduzindo a capacidade para o trabalho e desenvolvendo doenças relacionadas ao trabalho. Nesse contexto, sujeitos que exercem atividades laborais com maior exigência mental tendem a ter sua capacidade para o trabalho mais preservada do que aqueles que têm exigências mais físicas ${ }^{25}$.

Em um estudo com indivíduos com dor cervical, foi relatado que trabalhadores que estavam mais expostos a exigências físicas e duradouras provocavam estresse nos sistemas cardiovascular e musculoesquelético, o que estava relacionado a um maior grau de incapacidade ou desvantagem ${ }^{26}$. Essa poderia ser uma explicação para a associação encontrada no presente estudo entre a capacidade para o trabalho e o domínio dor física do OHIP-14, mesmo após ter sido controlada por variáveis sociodemográficas e de autopercepção.

As evidências sugerem que a qualidade de vida relacionada à saúde é multidimensional, incluindo dimensões físicas, sociais, psicológicas, desvantagens ou limitações, sendo o OHIP 
capaz de mensurá-las ${ }^{27}$. Esse indicador contribui para melhor esclarecer o "impacto social" das doenças bucais e pode ser útil para planejar ações para a saúde bucal dos trabalhadores. Além disso, essa abordagem permite identificar o presenteísmo, em que, apesar de não se afastar de sua atividade laboral, o desempenho, a segurança e o bem-estar do empregado são reduzidos ${ }^{28}$.

O estado de saúde bucal também pode ser afetado pelas consequências das exigências e das circunstâncias do trabalho. Na verdade, tanto as circunstâncias do ambiente de trabalho quanto o estado de saúde bucal podem reduzir a capacidade de trabalho, o que significa que, quanto maior o tempo em que o trabalhador estiver exposto a algumas circunstâncias desfavoráveis, mais sua capacidade de trabalho será afetada ${ }^{21,29}$.

A capacidade para o trabalho foi associada independentemente com a autopercepção de saúde bucal, particularmente no domínio dor física, o que justificaria ações de promoção, proteção e recuperação de saúde bucal dirigida a trabalhadores. Essa evidência reforça a importância das práticas de saúde bucal como componente de programas de saúde ocupacional, a fim de integrar ações de vigilância. Informações educativas sobre a saúde bucal no ambiente de trabalho, assim como exames periódicos, também são de extrema importância não só para monitorar a saúde e o bem-estar, mas para integrar atividades ocupacionais e cuidados de saúde.

\section{REFERÊNCIAS}

1. Pizzatto E, Garbin CAS. Odontologia do trabalho: implantação da atenção de sáude bucal do trabalhador. Odontol Clín Cient. 2006;5(2):99-102.

2. Guerra MJC, Greco RM, Leite ICG, Ferreira EF, Paula MVQ. Impacto das condições de saúde bucal na qualidade de vida de trabalhadores. Cien Saude Colet. 2014 dez;19(12):4777-86. http://dx.doi.org/10.1590/1413812320141912.21352013. PMid:25388186.

3. Abbas I, Mohammad SA, Peddireddy PR, Mocherla M, Koppula YR, Avidapu R. Oral health status of underground coal mine workers of ramakrishnapur, adilabad district, telangana, india - a cross-sectional study. J Clin Diagn Res. 2016 jan;10(1):ZC28-31. PMid:26894171.

4. World Health Organization. The WHOQOL group 1995 - The World Health Organization quality of life assessment (WHOQOL): position paper from the World Health Organization. Soc Sci Med. 1995;41(10):1403-9. http://dx.doi.org/10.1016/0277-9536(95)00112-K. PMid:8560308.

5. Macedo IAB, Costa SS. Saúde bucal e sua influência na qualidade de vida do trabalhador: uma revisão de artigos publicados a partir do ano de 1990. Rev Bras Med Trab. 2015;13(1):2-12.

6. Noronha DD, Martins AMEBL, Dias DS, Silveira MF, Paula AMB, Haikal DSA. Qualidade de vida relacionada à saúde entre adultos e fatores associados: um estudo de base populacional. Cienc Saude Colet. 2016;21(2):463-74. http://dx.doi.org/10.1590/1413-81232015212.01102015.

7. Brasil. Lei no 11.091 , de 12 de janeiro de 2005. Dispõe sobre a estruturação do Plano de Carreira dos Cargos Técnico-Administrativos em Educação, no âmbito das Instituições Federais de Ensino vinculadas ao Ministério da Educação, e dá outras providências. Diário Oficial da União, Brasília, 12 janeiro de 2005; Seção 1. p. 1.

8. Godinho MR, Greco RM, Teixeira MTB, Teixeira LR, Guerra MR, Chaoubah A. Work ability and associated factors of Brazilian technical-administrative workers in education. BMC Res Notes. 2016;9(1):1-10. http://dx.doi. org/10.1186/s13104-015-1837-x. PMid:26725043.

9. Oliveira BHD, Nadanovsky P. Psychometric properties of the Brazilian version of the Oral Health Impact Profile-short form. Community Dent Oral Epidemiol. 2005 ago;33(4):307-14. http://dx.doi.org/10.1111/j.16000528.2005.00225.x. PMid:16008638.

10. Martinez MC, Latorre MRDO, Fischer FM. Validade e confiabilidade da versão brasileira do índice de Capacidade para o Trabalho. Rev Saude Publica. 2009;43(3):525-32. http://dx.doi.org/10.1590/S0034-89102009005000017. PMid:19347177.
11. Tuomi K, Ilmarinen J, Seitsamo J, Huuhtanen P, Martikainen R, Nygård $\mathrm{CH}$, et al. Summary of the Finnish research project (1981-1992) to promote the health and work ability of aging workers. Scand J Work Environ Health. 1997;23(Suppl 1):66-71. PMid:9247997.

12. Andrade MV, Noronha KVMS, Menezes RM, Souza MN, Reis CB, Martins DR, et al. Desigualdade socioeconômica no acesso aos serviços de saúde no brasil: um estudo comparativo entre as regiões brasileiras em 1998 e 2008. Econ Apl. 2013;17(4):623-45. http://dx.doi.org/10.1590/S141380502013000400005

13. Macedo CG, Queluz DP. Quality of life and self-perceived oral health among workers from a furniture industry. Braz J Oral Sci. 2011;10(4):226-32.

14. Acharya S, Pentapati KC. Work stress and oral health-related quality of life among Indian information technology workers: an exploratory study. Int Dent J. 2012;62(3):132-6. http://dx.doi.org/10.1111/j.1875-595X.2011.00104.x. PMid:22568737.

15. Patil VV, Shigli K, Hebbal M, Agrawal N. Tooth loss, prosthetic status and treatment needs among industrial workers in Belgaum, Karnataka, India. J Oral Sci. 2012;54(4):285-92. http://dx.doi.org/10.2334/josnusd.54.285. PMid:23221153.

16. Marinho VC. Applying prescription-strength home-use and professionally applied topical fluoride products may benefit people at high risk for caries - the American Dental Association (ADA) 2013 clinical practice guideline recommendations. J Evid Based Dent Pract. 2014;14(3):120-3. http://dx.doi. org/10.1016/j.jebdp.2014.07.011. PMid:25234211.

17. Sanders AE, Slade GD, Lim S, Reisine ST. Impact of oral disease on quality of life in the US and Australian populations. Community Dent Oral Epidemiol. 2009 abr;37(2):171-81. http://dx.doi.org/10.1111/j.1600-0528.2008.00457.x. PMid:19175659.

18. Locker D. Deprivation and oral health: a review. Community Dent Oral Epidemiol. 2000;28(3):161-9. http://dx.doi.org/10.1034/j.16000528.2000.280301.x. PMid:10830642.

19. Mendonça BMC, Cimões R, Araújo ACS, Caldas Jr AF, Silva PV. Impacto do número de dentes presentes no desempenho de atividades diárias: estudo piloto. Cienc Saude Colet. 2010;15(3):775-84. http://dx.doi.org/10.1590/ S1413-81232010000300020.

20. Sio S, Cedrone F, Sanità D, Ricci P, Corbosiero P, Traglia M, et al. Quality of life in workers and stress: gender differences in exposure to psychosocial 
risks and perceived well-being. BioMed Res Int. 2017;2017: 7340781. http:// dx.doi.org/10.1155/2017/7340781. PMid:29349081.

21. Bomfim RA, Crosato E, Mazzilli LEN. Relations between oral health and work ability among administrative workers. Braz J Oral Sci. 2015 jan/ mar;14(1):1-5. http://dx.doi.org/10.1590/1677-3225v14n1a09.

22. Gift HC, Atchison KA, Dayton CM. Conceptualizing oral health and oral health-related quality of life. Soc Sci Med. 1997 mar;44(5):601-8. http:// dx.doi.org/10.1016/S0277-9536(96)00211-0. PMid:9032828.

23. Karlsson ML, Busch H, Aboagye E, Jensen I. Validation of a measure of health-related production loss: construct validity and responsiveness - a cohort study. BMC Public Health. 2015;15(1148):1-10.

24. Kotzer RD, Lawrence HP, Clovis JB, Matthews DC. Oral health-related quality of life in an aging Canadian population. Health Qual Life Outcomes. 2012;10:50. PMid:22587387.

25. Martinez MC, Latorre MRD. Saúde e capacidade de trabalho em trabalhadores de área administrativa. Rev Saude Publica. 2006;40(5):851-8. http://dx.doi. org/10.1590/S0034-89102006000600015. PMid:17301907.
26. Oliv S, Noor A, Gustafsson E, Hagberg M. A lower level of physically demanding work is associated with excellent work ability in men and women with neck pain in different age groups. Saf Health Work. 2017 dez;8(4):35663. http://dx.doi.org/10.1016/j.shaw.2017.03.004. PMid:29276634.

27. Vettore MV, Aqeeli A. The roles of contextual and individual social determinants of oral health-related quality of life in Brazilian adults. Qual Life Res. 2016 abr;25(4):1029-42. http://dx.doi.org/10.1007/s11136-0151118-0. PMid:26342929.

28. Montero J, Bravo M, Vicente MP, Galindo MP, López JF, Albaladejo A. Dimensional structure of the oral health-related quality of life in healthy Spanish workers. Health Qual Life Outcomes. 2010;8:24. PMid:20170546.

29. Lacerda JT, Ribeiro JD, Ribeiro DM, Traebert J. Prevalência da dor orofacial e seu impacto no desempenho diário em trabalhadores das indústrias têxteis do município de Laguna, SC. Cien Saude Colet. 2011;16(10):4275-82. http:// dx.doi.org/10.1590/S1413-81232011001100033. PMid:22031156.

Recebido em: Mar. 21, 2018 Aprovado em: Nov. 19, 2018 